

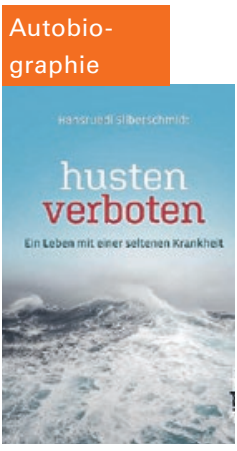

\section{husten verboten}

Hansruedi

Silberschmidt

Zürich: elfundzehn; 2021

Der kleine Hansruedi, geboren 1958, leidet seit seiner Geburt an wiederkehrenden Atemwegsinfekten, Mittelohrentzündungen und unerklärlichen Fieberschüben. Für seine Eltern und die Ärzte sind die Symptome ein Rätsel. Bereits als Kind wird er von Arzt zu Arzt geschoben. Er soll genesen unter dem strengen Regime der Schwestern im Kindersanatorium in Davos, erhält schmerzhafte experimentelle Behandlungen, wird für Kuraufenthalte ans Meer geschickt. Trotzdem verbessert sich sein Zustand nicht. Er wird einfach nicht gesund. Erst mit 39 Jahren erhält er eine Diagnose.

Hansruedi Silberschmidt schildert in dieser Autobiographie eindrücklich sein Leben mit einer lange undiagnostizierten Erkrankung und den Leidensweg bis zur Diagnose Primäre Ciliäre Dyskinesie. Darüber hinaus thematisiert das Buch düstere Kapitel der Schweizer Pädiatriegeschichte, zum Beispiel wie Kinder von ihren Eltern getrennt monatelange unnütze Kuraufenthalte über sich ergehen lassen mussten. Trotz dieser Erfahrungen klagt Silberschmidt in seinem Buch nicht an, sondern schildert ehrlich, spannend und kurzweilig. Uns Ärztinnen und Ärzten gibt er mit diesem Buch die Möglichkeit, unser Tun kritisch zu hinterfragen. Denn trotz seiner typischen Symptomatik hat ihn jahrzehntelang niemand wirklich ernst genommen und an diese Erkrankung gedacht. Viele seltene Erkrankungen werden auch heutzutage erst spät oder gar nicht diagnostiziert. Hoffentlich geht uns Hansruedi Silberschmidts Geschichte durch den Kopf, wenn wir wieder eine Patientin mit unklaren chronischen Symptomen vor uns haben. Der Autor jedenfalls hat es geschafft, als informierter Patient mit seinem Engagement nun auch anderen Patientinnen und Patienten und nicht zuletzt uns Ärztinnen und Ärzten zu helfen.

Dr. med. Leonie Schreck, PhD-Studentin, Universität Bern

leonie.schreck[at]ispm.unibe.ch

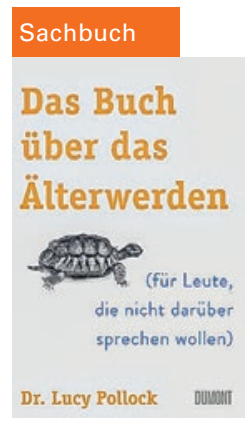

\section{Das Buch über das Älterwerden Lucy Pollock}

Köln: Dumont Verlag; 2021

«Wenn ich auf meine Jahre als Geriaterin zurückschaue, dann wird mir klar, dass meine Patient*innen eine Bereicherung gewesen sind, dass sie auf jeden Fall 'etwas zu geben' hatten, denn ich bin die Empfängerin ihrer Gaben gewesen.» Dieses Fazit zieht die Ärztin Lucy Pollock in ihrem kürzlich auch auf Deutsch erschienenen Buch. Die Britin gewährt den Lesenden Einblicke in ihren beruflichen Alltag als Fachärztin für Geriatrie. Dabei geht Pollock in 18 Kapiteln auf verschiedene Aspekte, mit denen sich ältere Menschen und ihre Angehörigen häufig konfrontiert sehen, ein: von Fahruntüchtigkeit, Inkontinenz und Impotenz über falsch dosierte Medikamente und Polymorbidität bis hin zum oft steinigen Weg in die Demenz. Diese Themen illustriert Pollock anhand von Fallbeispielen, welche sie einerseits mit aktuellen wissenschaftlichen Erkenntnissen ergänzt. Andererseits beschreibt die Fachärztin aber auch die verschiedenen Metaebenen, welche mit den einzelnen Themenbereichen einhergehen, wie beispielsweise Schamgefühle und hinderliche Tabus im Gespräch mit Angehörigen. Das Ganze würzt Lucy Pollock mit viel Empathie und einer passenden Prise britischen Humors. Die Autorin möchte mit dem Buch älteren Personen, ihren Angehörigen, aber auch den behandelnden Ärztinnen und Ärzten Mut machen, einen offenen respektvollen Dialog zu führen - egal wie heikel das Thema ist. Denn: Älter werden wir alle. Also lasst uns auch darüber sprechen und den Alterungsprozess mit gegenseitiger Wertschätzung und Realismus angehen.

Matthias Scholer, Chefredaktor SÄZ

matthias.scholer[at]emh.ch

\section{Teilen Sie Ihre literarischen Entdeckungen mit uns!}

Sie haben ein interessantes Buch gelesen und möchten es einem weiteren Leserkreis vorstellen?

Schicken Sie uns Ihre Buchbesprechung (max. 1200 Zeichen inkl. Leerzeichen) an: redaktion.saez[at]emh.ch

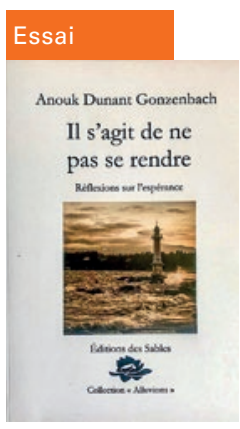

Il s'agit de ne pas se rendre

Anouk Dunant

Gonzenbach

Genève: Ed. des Sables; 2020

A l'été 2006, alors qu'elle est en vacances en Engadine avec son mari et sa fille, Anouk Dunant Gonzenbach doit interrompre sa grossesse alors avancée de cinq mois. Un petit trou a perforé la poche des eaux. Pourquoi ce trou s'est formé, les médecins ne le savent pas. Passé le choc et la tristesse - voire le désespoir -, l'auteure genevoise tente de recomposer le puzzle et de comprendre cette épreuve brutale et douloureuse. Elle est assaillie par d'innombrables questions. Des questions qui restent sans réponse. Comment se remettre de la perte de son enfant à ce stade de la grossesse? Pourquoi est-ce arrivé? Pourquoi devoir vivre un tel drame? Pourquoi n'a-t-on rien vu venir, malgré les possibilités de la médecine moderne? Comment accepter qu'il n'y ait pas d'explication médicale plausible? Avec les années, ce bouillonnement de pensées intérieures et les dizaines de pages griffonnées mènent Anouk Dunant Gonzenbach à entamer une réflexion introspective et spirituelle. Archiviste de métier, elle explore des pistes s'appuyant sur l'histoire, la littérature, la poésie et la religion. Davantage que le deuil périnatal, cet ouvrage met avant tout en lumière les notions d'espoir et de foi avec beaucoup de sensibilité, de justesse, d'authenticité et de profondeur, poussant la lectrice et le lecteur à se questionner sur sa propre compréhension de l'espérance. Qu'est-ce qui nous fait espérer et avancer chaque jour malgré les aléas de la vie? Qu'est-ce qui compte vraiment dans notre existence?

Julia Rippstein, rédactrice BMS

julia.rippstein[at]emh.ch

\section{Partagez vos découvertes littéraires!}

Vous avez lu un ouvrage intéressant et souhaitez en faire profiter d'autres lecteurs? Envoyez-nous votre critique littéraire (max. 1200 signes, espaces compris) à: redaction.bms[at]emh.ch 